\title{
Rotura del seno de Valsalva: Reporte de caso
}

\author{
Valsalva sine rupture: A case report \\ Martín Abel Paredes-Muñoz ${ }^{1, c}$, Héctor Alberto Montoya-Rojas ${ }^{1, \mathrm{c}}$, Francis Robert Pino- Zavaleta ${ }^{1, c ; 3}$, Víctor \\ Manuel Ponce-Mendoza ${ }^{1, \mathrm{c}}$, Jhunior Darwin Heber Reyes-Chávez ${ }^{1, \mathrm{c}}$, Luis Arturo Camacho-Saavedra ${ }^{2, a, b}$
}

\section{RESUMEN}

El aneurisma del seno de Valsalva es una entidad poco frecuente, con una incidencia de 0,09 a $0,15 \%$, en algunos casos los pacientes se presentan asintomáticos siempre que el aneurisma se encuentre íntegro. Si se perfora, se manifiesta como un cuadro de insuficiencia cardiaca rápidamente progresiva. Comunicamos el caso de una paciente de 27 años sin comorbilidades, con rotura del aneurisma del seno de Valsalva y se realiza una revisión bibliográfica del tema.

PALABRAS CLAVE: Aneurisma roto, mujeres embarazadas, preeclampsia. (Fuente: DeCS BIREME)

\section{SUMMARY}

Valsalva sinus aneurysm in an infrequent entity, the incidence is 0.09 to $0.15 \%$, most of the patients are asymptomatic while the aneurysm is intact. A rapidly progressive heart failure follows the perforation of it. We report here the case of a 27 -year-old male patient presenting with a rupture Valsalva sinus aneurysm and performed a literature review.

KEYWORDS: Aneurysm, ruptured, pregnant women, pre-eclampsia. (Source: MeSH NLM).

\section{INTRODUCCIÓN}

El seno roto de Valsalva es una entidad poco frecuente (1), representa $<1 \%$ de todos los defectos congénitos, se presenta entre los 13 y 65 años y predomina en el sexo masculino con una relación de $4: 1(2)$.

Se produce por dilatación en la unión de la capa media aórtica con el anillo fibroso de la válvula aórtica, y puede evolucionar a la ruptura o la infección (3). Si se perfora, se manifiesta como un cuadro de insuficiencia cardiaca rápidamente progresiva; además puede existir obstrucción al flujo en el tracto de salida ventricular derecha, insuficiencia aórtica, trastornos del ritmo y, raramente, isquemia miocárdica o necrosis (4); en ocasiones puede conducir a una muerte súbita secundaria a ruptura abierta a pericardio, infarto del miocardio o bloqueo aurículo-ventricular. La perforación puede ser secundaria a una endocarditis infecciosa injertada en el aneurisma del seno de Valsalva, donde la infección provoca la ruptura del seno causando insuficiencia cardiaca congestiva aguda y la muerte (5); sin embargo, en algunos casos los pacientes con aneurisma se presentan asintomáticos siempre que el aneurisma se encuentre íntegro.

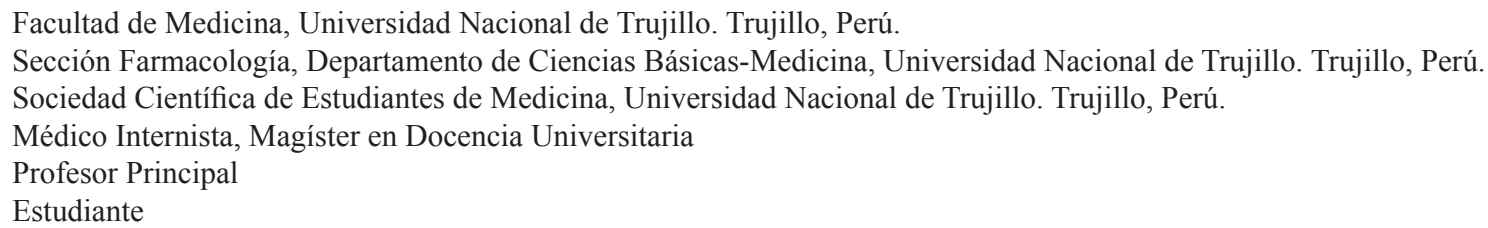


Frente a la rotura del aneurisma del seno de Valsalva el tratamiento de elección es la intervención quirúrgica $(1,2)$.

Siendo una enfermedad poco común y que no ha sido reportado en nuestro medio, se presenta el caso de una paciente joven con rotura de un aneurisma del seno de Valsalva.

\section{PRESENTACIÓN DEL CASO}

Mujer de 27 años sin comorbilidades, con antecedente de síndrome de HELLP asociado a embarazo gemelar y presencia de soplo holosistólico de grado 3/6 en foco mitral irradiado a axila izquierda de hace 5 años.

La paciente refería que desde un mes antes presentaba dolor precordial de tipo opresivo, leve sin irradiación y que cedía espontáneamente. Un día antes del ingreso, presentó palpitaciones y nuevamente dolor precordial de intensidad moderada sin irradiación, asociado a angustia.

La exploración al ingreso evidenció leve palidez y pulso célere; la auscultación cardiaca revelaba tonos rítmicos con frecuencia de $75 \mathrm{lat} / \mathrm{min}$, soplo sistólico de grado $3 / 6$ en foco mitral con irradiación hacia la axila, no se percibió choque de punta. El electrocardiograma mostró QT prolongado de $470 \mathrm{~ms}$ y el ecocardiograma evidenció dilatación de las cuatro cavidades cardiacas, esclerosis y engrosamiento

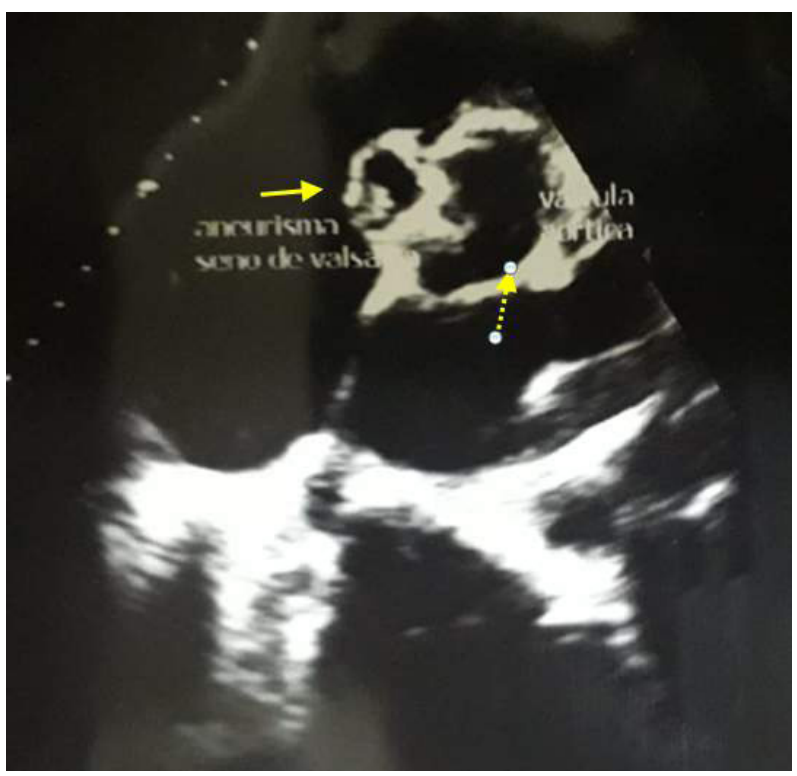

Figura 1. Ecocardiograma en el que se aprecia aneurisma del seno de Valsaba (flecha continua) y engrosamiento de las válvulas aorticas (flecha discontinua). valvular aórtico con lesión nodular adyacente correspondiente a aneurisma del seno de Valsalva que se encontraba protruida hacia la aurícula derecha y perforada, formando una comunicación con la cavidad mencionada (figura 1). También reveló insuficiencia aórtica severa, insuficiencia mitral leve, insuficiencia tricuspídea severa e hipertensión pulmonar con una presión de $50 \mathrm{~mm} \mathrm{Hg}$.

Se solicitó interconsulta al servicio de cirugía de tórax para realizar el tratamiento quirúrgico; sin embargo, dos días después del ingreso, encontrándose clínicamente estable, la paciente solicitó su alta voluntaria.

\section{DISCUSIÓN}

Se presenta un caso poco frecuente de una paciente de 27 años con aneurisma en seno de Valsalva (SOVA) roto, de inusual presentación a esta edad pues generalmente la ruptura ocurre en varones mayores de 40 años (2).

Tenía antecedente de embarazo gemelar y síndrome HELLP de 5 años antes, asociando a pre eclampsia. Estos dos procesos influyen en el desarrollo de la enfermedad. El embarazo produce cambios hemodinámicos (aumento del volumen circulante, aumento de la frecuencia cardiaca, aumento del gasto cardíaco) que sirven para cumplir las demandas metabólicas (6). Estos cambios generados por el embarazo pueden aumentar el volumen sanguíneo total generando una dilatación en el diámetro de la aorta perjudicando el estado cardíaco (7), siendo mayor en condiciones de hipertensión arterial como ocurre en la pre eclampsia, causando condiciones inestables en la pared arterial que pueden llegar a producir un aneurisma.

La formación de este aneurisma se da de manera asintomática y la ruptura, que es el evento más común, puede ocurrir de manera espontánea, por trauma, ejercicio físico o infección (6). Los aneurismas no rotos suelen ser asintomáticos o presentar síntomas como: disnea, angina, arritmias por fibrilación auricular o bloqueo cardiaco completo. La ruptura tiene consecuencias que dependen de su velocidad, ubicación y tamaño.

La ruptura del seno derecho es el más frecuente $(60 \%)$ y peligroso pues genera una comunicación de aorta con el tracto de salida del ventrículo derecho $(60 \%)$, con el atrio derecho (30\%), tal como sucedió 
en el presente caso o con la aurícula izquierda (10\%) (2). Se genera un cortocircuito de izquierda a derecha, con insuficiencia cardiaca congestiva aguda, que se manifiesta con insuficiencia aórtica aguda, soplo sistólico- diastólico de reciente inicio en muchos casos, disnea de leve a grave, taponamiento cardiaco, incluso muerte súbita $(2,8)$. Sin embargo; el caso presentado cursó con una evolución lenta, esto podría deberse a la formación de la fístula hacia la aurícula derecha, por lo que podría pasar inadvertido por varios años, hasta que un evento origine la rotura del aneurisma, como por ejemplo, endocarditis bacteriana o un evento de estrés.

Dada la sintomatología como el soplo sistólico y la insuficiencia aórtica se sospechó de un problema estructural de origen cardiaco y se le realizó un estudio de ecocardiografía estándar por su alta especificidad y sensibilidad, en el cual se pudo evidenciar el aneurisma del seno de Valsalva.

El tratamiento recomendado para un SOVA roto, congénito o agrandado es la intervención quirúrgica urgente que normalmente tiene un bajo riesgo de mortalidad $(2,8,9)$.

\section{Declaración de conflictos de interés:}

Ninguno.

Contribución de autoría:

MAPM, HAMR, FRPZ, VMPM, JDHRC, LACS: Concepción y diseño del reporte de caso, recolección de datos del paciente, análisis e interpretación de datos; redacción del reporte; revisión crítica del artículo; aprobación de la versión final; aporte de material de estudio.

\section{Correspondencia:}

Martin Abel Paredes-Muñoz

Correo electrónico: martinabel250397@gmail.com

\section{REFERENCIAS BIBLIOGRÁFICAS}

1. González O, Torres J, Hernández J. Aneurisma del seno de Valsalva roto con fístula a ventrículo derecho. Presentación de un caso clínico y revisión de la literatura. Insuf Card. 2017; 12(3):134-141.

2. Cao L, Hannon D, Movahed A. Rotura del seno de Valsalva no coronario en la aurícula derecha con una comunicación interventricular perimembranosa existente. World J Clin Cases. 2013; 1(4):146-148.

3. Malkum C, Ruiz F, Muñoz A, Heilbron O, López R. Aneurisma del seno de Valsalva derecho roto disecado al septum ventricular. Rev Colomb Cardiol. 2019; 26(3):151-152.

4. Jiménez J, Montoya J, Valdespino A, et al. Recurrent aneurysm of sinus of Valsalva, the world's first case report. Rev Med Inst Mex Seguro Soc. 2015; 53(6):732-5.

5. Rendon J, Duarte N. Aneurisma del seno de Valsalva roto. Presentación de un caso evaluado con ecocardiografía tridimensional en tiempo real. Rev Colomb Cardiol. 2011; 18(3):154-157.

6. Zavala-Barrios B, García-Castanedo C, Viruez Soto J, Briones-Garduño J, Coronel-Cruz F. Aneurisma de aorta ascendente, hipertiroidismo y embarazo. Caso clínico. Ginecol Obstet Mex. 2015; 83:627-634.

7. Latzman J, Makaryus AN, Rosman D. Ruptured Sinus of Valsalva aneurysm in a pregnant woman. Tex Heart Inst J. 2006; 33(1):66-69.

8. Charfeddine S, Abid D, Triki F, Abid L, Kammoun $\mathrm{S}$, Frikha I. Unusual case of ruptured sinus of valsalva aneurysm in a pregnant woman. Pan Afr Med J. 2017; 27:271. DOI: 10.11604/pamj.2017.27.271.9741

9. Weinreich M, Yu P, Trost B. Sinus of Valsalva aneurysms: Review of the literature and an update on management. Clin Cardiol. 2015; 38 (3):185-189. DOI: $10.1002 /$ clc. 22359 